\title{
Sobre el progreso en una era de revolución científico-tecnológico-digital
}

\author{
Nùria Almiron \\ Profesora de Políticas de Comunicación \\ Universidad Autónoma de Barcelona
}

\section{RESUMEN}

El presente artículo bucea en las fuentes de la idea de progreso hasta situarla en la actualidad, momento en el que se plantea la paradoja del pesimismo y la irracionalidad reinantes en una era de desarrollo tecnológico y científico sin precedentes. El choque entre la euforia determinista (el desarrollo tecnológico comportaría automáticamente desarrollo social) y el escepticismo irracional frente a la ciencia (a pesar del progreso técnico lo que reinaría es la decadencia moral en plena sociedad del conocimiento) es el objeto de análisis de este artículo.

\begin{abstract}
In this paper the author looks back over the idea of progress sources from the past to the present, when a paradox emerges: pessimism an irrationality arise in an era of tremendous technological and scientific development. The collision between the deterministic euphoria (which states that technological development produces automatic social development) and the irrational scepticism towards the science (which states that, in spite of all the technical progress, moral decadence is the king in our society of knowledge) produces a clash which is the purpose of this paper analyses.
\end{abstract}

Palabras claves: Progreso tecnológico/Progreso social/Contradicciones/Era digital.

Key words: Tecnological progress/Social progress/Contradictions/Digital era.

\section{Introducción}

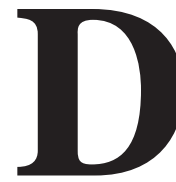

esde que nuestros ancestros no erectos empezaron a modificar el paisaje del planeta, hace ahora unos diez mil millones de años, hasta la posibilidad de clonación de la especie humana ha trascurrido un largo periodo de tiempo en el que el ser humano ha experimentado una transformación biológica, social e intelectual gigantesca.

A nadie razonable le cabe la menor duda que hoy vivimos mucho mejor, en nuestros artificiales hogares de ladrillos, cristal y cemento, que cuando habitábamos las oscuras y húmedas cuevas del neolítico. No sólo somos mucho más longevos, porque nos alimentamos mejor y nos desgastamos menos, sino que 
además hemos aprendido a disfrutar de este intervalo de tiempo entre el nacimiento y la muerte que es la existencia. El bienestar y el placer han sustituido a la lucha por la mera supervivencia fisiológica. Y si lo anterior no es cierto para todos, no lo es menos que debería serlo, porque el potencial para ello existe. Desafortunadamente, no todos los individuos del planeta llevan una vida cómoda y fácil, especialmente aquellos que no habitan en las regiones privilegiadas del mismo (en realidad, y para oprobio nuestro, una aplastante mayoría), pero incluso los que en el siglo XXI padecen las condiciones de vida más infames experimentan una situación comparativamente mejor a la de nuestros antepasados de Altamira. En realidad, que su vida era mejor que la de los primeros humanos es algo que ya pensaban hasta los griegos de la Antigüedad, no ha hecho falta llegar al segundo milenio de esta era para descubrirlo y tecletearlo en un ordenador portátil ultraligero fabricado a base de silicio y titanio (por cierto, los dos grandes iconos de la presente era).

Hace más de 2700 años, cuando Grecia todavía vivía en su época arcaica y el analfabetismo reinante en la región hacía de la transmisión oral la primera y principal tecnología de la información, un poeta-filósofo como Hesiodo (el que difundiera el mito de Pandora, el gran competidor dicen de Homero, si es que Homero jamás existió) ya planteaba la idea de progreso, esto es, la diferencia de su situación con la de los tiempos primitivos y, asímismo, su diferencia con los tiempos venideros (Hesiodo ya creía que si se imponía la justicia, algo que sólo podría hacerse por medio de reformas sociales y de un esfuerzo individual constantes, el futuro sería mejor). Tal y como recuerda Robert Nisbet en Historia de la idea de progreso (1980), Hesiodo no sería el único en plantear esta idea, tras él personajes como el autor teatral Esquilo, el sofista Protágoras o el historiador Tucídides contribuyen igualmente a forjar, ímplicita o explícitamente, la idea de que el presente es superior al pasado y que cabe esperar que el futuro sea mejor que el presente. Nisbet incluso incluye a Platón, que para muchos es totalmente ajeno a la idea de cambio, pues también este filósofo griego habla en algunas de sus obras (por ejemplo en Las leyes) del desarrollo de la humanidad y del progreso de las instituciones a lo largo de grandes períodos de tiempo.

No es menos cierto que en la Grecia antigua también encontramos la noción, más relevante para muchos y totalmente contraria a la idea de progreso, de una edad de oro anterior, una Arcadia feliz que mitificada y utopizada por el pensamiento occidental enlaza con el elogio del "primitivismo" y del buen salvaje (la idealización de la figura del ser primitivo como un individuo noble, todavía sin corromper por la sociedad). Esta idea del "buen salvaje", nacida incluso antes de Platón, se transmitirá casi inalterable a través de las etapas griega y romana, el periodo helenista, el Renacimiento y el Barroco, y seguirá vigente en el pensamiento occidental de la Ilustración (recuperada con fuerza por Rousseau) y del 
Romanticismo hasta hoy. Pero que esta idea estuviera muy extendida no invalida que autores como Robert Nisbet sitúen la aparición de la noción de progreso hace ahora casi 3.000 años.

Es más, este sociólogo norteamericano intenta demostrar, en ocasiones con bastante éxito, que esta idea no sólo es premoderna sino que además es una constante que no nos abandona desde su nacimiento y que tiene momentos muy álgidos incluso antes de alcanzar su cenit en el siglo XVIII y XIX. La idea de progreso llega así a convertirse en un dogma para la humanidad, al menos hasta la llegada del siglo XX. Tal fe en el progreso es significativa porque transmite no sólo que hemos avanzado constantemente desde todos los puntos de vista, sino que, además, la idea de progreso nos ha impulsado a progresar. Antes de que estallara la primera guerra mundial y se sucedieran toda la serie de conflictos que convirtieron al siglo XX en el más sangriento de la historia de la humanidad (nada puede igualarse a los 50 millones de muertos habidos sólo en las dos grandes contiendas mundiales del siglo pasado), los habitantes de este planeta estaban mayoritariamente convencidos de su superioridad con respecto a sus predecesores. Esto es, creían que habían avanzado mucho a nivel científico y tecnológico y que ello les había traído más bienestar social. Consideraban que esa mayor calidad de vida les había comportado un progreso moral e intelectual. Sin embargo, los comportamientos de algunos de esos mismos seres humanos a lo largo del siglo XX y el revisionismo de la historia de la expansión de Occidente (que de azaña memorable ha pasado a ser considerada con un sentimiento de culpabilidad) restarían bastantes miembros a la comunidad de creyentes en el progreso.

Hoy en día, a comienzos de un siglo XXI lleno de todo tipo de premoniciones enfrentadas (como las derivadas de la promesa biotecnológica) y con lo que algunos han llamado la "aventura de la Ilustración" ya zanjada (los ideales de la Ilustración no han desaparecido pero está claro que no han triunfado), siguen existiendo muchas personas que creen en el progreso de la humanidad. Para ellos no se explica de otro modo que la civilización haya conseguido sobrevivirse a sí misma a pesar de todas las crisis por las que ha pasado y consideran las etapas de retroceso como fases de decadencia necesarias para que se produjera un nuevo y cada vez más brutal relanzamiento.

\section{Los hitos informacionales de la humanidad}

Una ojeada a los logros de la humanidad evidencia que de relanzamientos ha habido muchos. Siguiendo a Michael Rothschild (1990) podríamos decir que la humanidad ha pasado hasta hoy por al menos seis grandes revoluciones que se corresponden con lo que algunos han llamado certeramente "explosiones informacionales". Cada nuevo paso dado por la sociedad es fruto de una información previamente adquirida y convertida en conocimiento, y produce a su vez más información nueva. 
La primera explosión informacional data de hace unos 17.000 millones de años, acaso esto es lo mínimo que se puede decir de las primeras pinturas rupestres de las que tenemos conocimiento: las de las cuevas de Altamira. Cuando el hombre primitivo empieza a manchar las paredes de sus hábitats con símbolos que narran historias, la humanidad está tomando un camino sin retorno. La siguiente revolución tiene lugar ya en la protohistoria (entre el 3100 y el 2700 a.c.) y se produce a raíz de la invención de la escritura por parte de los sumerios. La escritura cuneiforme nace fruto de la necesidad que tiene Sumer de gestionar con eficacia su información económica (de registrar sus negociaciones comerciales con otras regiones distantes) y es además la máxima expresión de una cultura engendradora de civilizaciones sólo comparable a la generada siglos después por la Grecia clásica. Después de estas épocas no encontramos otra explosión informacional que merezca el apelativo de revolucionaria hasta el siglo $\mathrm{XV}$, gracias al invento de la imprenta de tipos móviles de un tal Johannes Gensfleisch, más conocido como Gutenberg. La aparición de la impresión mecanizada a partir de 1455 reduciría en un $98 \%$ la mano de obra necesaria para copiar información. A continuación, el siglo XVII y el siglo XVIII verían el nacimiento de la ciencia moderna (lo cual no significa que antes no existieran conocimientos científicos sino que no existía ciencia ni método científico tal y como los definimos hoy, véase: Popper, 1962; Díez y Ulises, 1999; o Habermas, 1988) y constituirían un nuevo periodo de grandes cambios para la humanidad. En el siglo XIX, la revolución industrial cambiaría de nuevo por completo la forma de concebir (y de polucionar) el mundo. Algunas zonas del planeta todavía no han realizado hoy en día esta transformación y es probable que sobrevivan sin experimentarla porque están dando el salto directo a la última explosión de conocimiento, la tecnológicodigital. La revolución de la microinformática recibe, para algunos con todos los honores, el apelativo de "informacional", porque en ella la información se convierte más que nunca en el bien más preciado. Como afirma el sociólogo Manuel Castells (1997), la información no es ya sólo fuente de conocimiento, herramienta, sino objeto de valor en sí misma, participa de forma determinante en los procesos de generación de capital, esto es: la información ya no sólo es potencial de poder en abstracto, es poder bien concreto, poder económico.

Como han expresado perfectamente numerosos autores (por ejemplo el mismo Rothschild), la presente explosión informacional se caracteriza por manipular la información principalmente en dos formas muy novedosas, genética y binaria, y por conquistar los logros más espectaculares en amplitud (cantidad de personas afectadas) y en profundidad (calidad o alcance del cambio material aportado) de la historia de la humanidad. Desde la segunda guerra mundial del siglo XX, hace ahora más de 50 años, hasta la actualidad, la revolución científicotecnológica-digital ha puesto en nuestras manos una enorme cantidad de sorpresas. Es cierto que no se han cumplido todos los sueños de la ciencia ficción literaria, 
pero también es cierto que muchas de las actuales innovaciones ni siquiera habían sido anticipadas. Dentro del ámbito de las no cumplidas están predicciones muy serias realizadas por especialistas: no hemos sustituido el petróleo y la electricidad por nuevas fuentes de energía, no hemos reducido los defectos hereditarios y congénitos, las técnicas ciborg (implantación de órganos artificiales para sustituir órganos humanos defectuosos) son muy limitadas, no hemos logrado la hibernación humana, no controlamos la meteorología ni el clima (sólo podemos intentar predecirlos), no podemos comunicarnos directamente con el cerebro (sin emplear el habla), no hemos colonizado el espacio ni el mundo submarino, no hemos inventado plataformas volantes individuales para transportarnos (tan populares en las películas futuristas), ni siquiera usamos masivamente los videoteléfonos (teléfonos con pantalla de televisión) que ya anticiparan las películas de hace más de treinta años. Estas y muchas otras predicciones incumplidas no corresponden sólo a la ciencia ficción cinematográfica, también las podemos encontrar en un famoso y serio libro de prospectiva titulado Hacia el año 2000 (1967) firmado por reputados expertos. Ahora bien, la realidad ha superado muchas de las espectativas de artículos como éste, especialmente en el ámbito de la microinformática.

El momento culminante que marca el simbólico disparo de salida a la nueva era se sitúa a finales de la década de los cincuenta con un disparo bien real y atronador: el del lanzamiento del primer satélite de comunicaciones espacial, el Sputnik, por parte de la extinta Unión Soviética. Mientras, casi simultaneamente, el presidente norteamericano Eisenhower da orden de crear una importante agencia de investigación a la que se dotará de enormes recursos y libertad para que los Estados Unidos no queden rezagados en la carrera tecnológica. Se trata de la famosa ARPA, el origen de la actual Internet. Luego fuimos a la Luna y redujimos el tamaño de los ordenadores de forma abismal al tiempo que multiplicamos su potencia exponencialmente, de forma que este libro ha podido ser escrito en un silfídeo artefacto de apenas 3 kilogramos de peso y 2 centímetros de grosor pero más potente que cualquiera de los ordenadores con que trabajaban los científicos del departamento de defensa norteamericano no 50 años atrás sino hace sólo una década. Por el camino hemos empezado a digitalizar el contenido del mundo, lo que significa que pronto podremos acceder a todo el conocimiento acumulado por la humanidad a través de cualquier nodo conectado a la Red planetaria en que se ha convertido Internet. Pero hay mucho más, la introducción de microprocesadores en cualquier utensilio, medio de transporte o máquina los convierte automáticamente en inteligentes, de modo que nos ponemos en manos de los ordenadores para volar a miles de metros de altura, regular la ingesta de medicamentos peligrosos, construir edificios, hacer negocios, gestionar nuestro dinero, divertirnos o manipular alimentos. La inteligencia artificial cada vez es capaz de robotizar más comportamientos y todos sabemos cuanto les cuesta a los grandes maestros del ajedrez ganar a los ajedrecistas de silicio. Y mientras implantamos chips bajo 
la piel de nuestros animales para no extraviarlos, la revolución del ADN hace que sea imposible que nos extraviemos nosotros. El día que descubrimos que llevamos nuestra identidad grabada en el núcleo de nuestras células nuestros actos adquirieron otra dimensión: cada uno de nosotros es único y nos es imposible eludir nuestra responsabilidad (Habermas, 2001).

Asi mismo, la transformación que hemos realizado en el campo y en la agricultura a lo largo de las últimas décadas no tiene parangón. Hemos multiplicado la resistencia de las semillas a todo tipo de plagas, al tiempo que generamos especies más y más productivas y las labores del campo han quedado en manos de unas pocas personas, a penas el 3\% o el $4 \%$ de la población en los países avanzados, porque la maquinaria y los productos químicos reducen el trabajo radicalmente. Con las mezclas genéticas vamos camino de lograr lo impensable: alimentar cada vez a más personas con menores extensiones de terreno y menores costes. Pero es en el campo de la genética humana donde, por supuesto, los avances en biotecnología son más impactantes. Se ha conseguido secuenciar el genoma humano tan rápido que todavía no sabemos qué significa el resultado obtenido de su desciframiento. Tenemos el código pero no sabemos qué secretos esconde. Aunque no tardaremos mucho en averiguarlo. Con esa información y los enormes avances realizados en los últimos años en el ámbito de la ingeniería genética es posible que podamos curar enfermedades hasta ahora consideradas incurables. Los progresos realizados en dolencias graves como el cáncer o el SIDA son ya en estos momentos inmensos. Hace sólo unos años recibir un diagnóstico de una de estas enfermedades significaba una probabilidad altísima de morir. Esa probabilidad se ha reducido drásticamente ahora, cuando no se ha invertido. Hoy en día la tecnología permite realizar operaciones complejas remotamente, allí donde no existen hospitales y sobrevivir a situaciones antes consideradas mortales. Y, por supuesto, se nos abre un abanico inmenso de nuevas posibilidades en campos como el tratamiento de las células madre. Si algunos se escandalizan con las madres/útero de alquiler, las abuelas/madre o las donaciones de semen anónimas, es de suponer que las promesas de la nueva bioingeniería no les dejarán incólumnes. La posibilidad de clonar partes de nuestro cuerpo (para sustituir órganos dañados sin rechazo), o seres humanos completos, y de diagnosticar preventivamente la calidad genética de los embriones que van a nacer (someterlos a una prueba genética cuando están en un estadio octocelular y no volverlos a implantar en el útero si no superan nuestras espectativas) no sólo aporta nuevas formas de curación, sino, también, dilemas morales y éticos inéditos. Algunos incluso ven posible la inmortalidad el día que, junto a los tejidos fisiológicos, seamos capaces de clonar/trasladar el cerebro con sus contenidos (y problemas), esto es, el alma. Para vivir eternamente bastaría así ir traspasando nuestra "materia intelectual" de cuerpo en cuerpo a medida que los "recipientes" fueran envejeciendo. 
En suma, la revolución científico-tecnológico-digital de los últimos años no sólo ha mejorado drásticamente nuestra esperanza y calidad de vida sino que también ha convencido a muchas personas de que con las nuevas tecnologías aumentaba su poder individual y colectivo. Al menos en teoría y en potencia somos seres mucho más proclives a entender el mundo (y, por lo tanto, a entendernos a nosotros mismos), a relacionarnos los unos con los otros y a vivir más extensa e intensamente que lo fueron cualquiera de nuestros antepasados. Para algunos esto significa que somos más proclives a ser felices. Pero ¿consiste en esto el progreso?

\section{Progreso social y progreso tecnológico}

Un tetrapléjico mudo que se comunica con el mundo a través de un ordenador en el que puede escribir gracias a un mecanismo sensible al parpadeo y a la mirada (esto es, al punto focal en el que se posan sus ojos en la pantalla) respondería indudablemente que sí a la pregunta anterior. Un campesino que trabaja completamente sólo centenares de hectáreas de duro campo probablemente también respondería afirmativamente, sin su tecnología biomecánica no podría sobrevivir, esto es, no conseguiría sacar el suficiente rendimiento al campo, necesitaría más manos y si tuviera que compartir con otros el fruto de la tierra ésta ya no le daría para vivir decentemente. También los habitantes de los poblados más míseros de Bangladesh, que no han conocido el teléfono fijo ni la máquina de escribir pero tienen teléfonos móviles y acceso a Internet, asentirían. Ello permite que sus hijos tengan alguna posibilidad de salir del círculo de la pobreza. Incluso un desarrollador de software cuyo programa de dosificación de anticoagulantes orales ayuda a salvar vidas cree en un futuro mejor. Todo esto es progreso en mayúsculas.

Sin embargo, mucha gente no comparte hoy en día esta opinión. En realidad cada vez menos gente la comparte. La cuestión radica en lo que consideramos o no consideramos progreso, esto es, en cómo vemos el cambio y en cómo valoramos sus efectos (algo que, lógicamente, tiene que ver directamente con la posición que cada uno de nosotros ocupa en la sociedad). Nos ayudará en la reflexión definir el concepto.

Por progreso entendemos casi siempre avance, mejora, adelanto. Y avance, mejora y adelanto son conceptos positivos. De hecho una "progresión" es la suma o multiplicación, la acumulación hacia delante, de cosas. Pero al progresar no sólo se va a más sino que también se va a mejor, hay un perfeccionamiento. El término comporta pues dos connotaciones distintas, una cuantitativa y otra cualitativa. Es evidente que hay personas que consideran que el cumplimiento de la primera connotación ya implica progreso porque automáticamente del progreso cuantitativo (material) se derivaría un progreso cualitativo (en el ámbito de la conciencia y la convivencia humanas, según la definición de Jürgen Habermas, 2001). Para 
otras, no es siempre evidente que de un progreso material se derive un perfeccionamiento moral. La relación actual entre ambas, cantidad/calidad del cambio, es una percepción engendrada en el siglo XVIII.

Entre 1750 y 1900 es cuando la idea de progreso alcanza su máximo apogeo. La concepción actual de progreso debe casi todo a este periodo. Tras la explosión informacional ocasionada por el nacimiento de la ciencia moderna en el siglo XVII, los intelectuales del Siglo de las Luces creyeron ver en los primeros descubrimientos científicos el camino para el perfeccionamiento máximo de la humanidad. Los hallazgos y las promesas de la ciencia otorgaron confianza ilimitada a la razón humana para conseguir una mejora indefinida a partir de ese momento. Gracias a la razón el hombre podía vencer a las fuerzas de la naturaleza e iniciar un camino ascendente en el que la idea de progreso ya no se desvincularía jamás, hasta hoy, de la ciencia y sus aplicaciones técnicas. La visión del universo quedaba así completamente alterada y no es para menos. Cuando se puede morir por una simple fiebre o, en el caso de las mujeres, por el mero hecho de sufrir un embarazo pasados los 40 años (prácticamente una condena a muerte todavía a finales del siglo XVIII), cualquier avance científico, por mínimo que sea, contagia de esperanza a los hombres. Los grandes avances de la ciencia no llegarán hasta el siglo XX, pero doscientos años antes la especie humana ya se convence que aquel camino, el de la ciencia y la razón, podía mejorar mucho su calidad de vida. La cosmovisión del ser humano queda definitivamente modificada cuando la ciencia le dota de poder (o le promete dotarle de ese poder) sobre su cuerpo y sobre la naturaleza.

A esta época se debe, además, otra asociación especialmente relevante para la idea de progreso. Es en este momento que a la noción de progreso se asocian ideas como las de libertad, igualdad y crecimiento económico, todas ellas hijas de la Ilustración (Nisbet, 1980). De ahí procede que hoy en día muchas personas vinculen inconscientemente que más desarrollo económico, más avance tecnológico y más modernización en general son sinónimos de progreso social.

Pero la Revolución Francesa también marca el punto de inicio a partir del cual la idea de progreso nos dividirá. Frente a los que pretenden primar la igualdad social se encontrarán aquellos para los que lo más importante es la libertad individual. La separación entre socialismo y liberalismo marcará la concepción de progreso tanto o más a como lo hará la gran ruptura filosófica de la historia del pensamiento occidental: la nueva filosofía de la existencia (existencialismo). La idea de una historia cíclica o no lineal, que se va repitiendo o se sucede en el tiempo sin que exista progresión evaluable de ninguna clase, o incluso produciendo un claro retroceso, es una noción que podemos rastrear hacia atrás hasta llegar a la Antigüedad, pero es Nietzsche (Hayman, 1980), fallecido en 1900, quien la recobra con más fuerza para el siglo XX. La vida no tiene sentido y es inútil buscárselo, afirmarán después los existencialistas ateos capitaneados por Jean Paul 
Sartre (Lévy, 2000). La felicidad no la encontraremos en una falsa idea de progreso y por primera vez puede decirse en voz bien alta que tampoco la encontraremos en Dios.

\section{El siglo XX: entre la irracionalidad y el determinismo tecnológico}

Llegados a la segunda mitad del siglo XX advertimos la consolidación de una disyuntiva radical en la valoración que los individuos hacen del presente y del futuro. Se trata de percepciones opuestas y que conducen a posiciones contrarias. Su coexistencia es desconcertantemente contradictoria. Se trata de la convivencia de dos enfoques que no son nuevos, están anclados en el pasado, pero que resurgen en el cambio de milenio con inusitada fuerza: son, por un lado, un reforzado determinismo tecnológico y, por otro, el auge de la irracionalidad.

El determinismo tecnológico es un viejo amigo del ser humano desde que la fe en la razón se instauró en los corazones de los ciudadanos de la Ilustración. A partir de ese momento, lo decía antes, se creó un vínculo inextricable entre la nueva religión, la ciencia, y el progreso social. La innovación tecnológica ha sido interpretada desde entonces por muchas personas como fuente de transformación social. Esto es, el cambio tecnológico aporta automáticamente cambio social o, lo que sería lo mismo, la principal causa del cambio social son las innovaciones tecnológicas. Desde este punto de vista, cuanto más revolucionaria es la nueva tecnología (más radicalmente innovadora con respecto a la anterior), mayor es el progreso social conseguido a través de ella. Esta visión de las cosas no es común sólo entre los grandes apasionados de la tecnología, los llamados tecnófilos, también lo es, curiosamente, entre sus grandes detractores, los tencnófobos (para una interesante reflexión sobre ambos ver: Graham, 2001) y muchos historiadores han establecido nexos directos entre la aparición de una determinada tecnología y un posterior cambio social: el molino y la sociedad feudal, la máquina de vapor y la sociedad industrial y, ahora, el microprocesador y la sociedad de la información. Sin embargo, en la inmensa mayoría de estas asociaciones, tal efecto directo no existe o, en todo caso, se prolonga a lo largo de un periodo muy extenso de tiempo. Es decir, si todas esas tecnologías tienen algun impacto en la sociedad, el resultado del mismo no se consolida hasta mucho tiempo después. No existe una causa-efecto inmediata por lo que es fácil suponer que habrá muchos otros elementos que participen de la transformación social posterior (Briggs y Burke, 2002).

Como describe Eduard Aibar (2001), el determinismo tecnológico es además un tópico estrechamente unido a la idea de la autonomía de la tecnología. Aquellos que piensan que a cualquier innovación tecnológica importante acaba siguiendo una innovación social lo hacen porque asocian a la tecnología una especie de fatalismo. Esto es, el desarrollo tecnológico se ve como algo imparable, inevitable, inexorable. Como si el desarrollo tecnológico fuera algo autónomo, 
unidireccional, que no podemos controlar. A lo largo de la década de los noventa del siglo pasado, la euforia desatada alrededor de las nuevas tecnologías de la información y la comunicación, abanderadas por Internet, llevó el determinismo tecnológico a sus cotas más altas en siglos. El crecimiento económico y el enriquecimiento rápido que se produjo alrededor de las telecomunicaciones y la red de redes hizo de este tópico verdad sacrosanta. El profesor Aibar afirma que ha llegado a formar parte del sentido común dar por supuesto que la revolución tecnológica de las telecomunicaciones va a suponer una transformación social. Lo cierto es que, durante la década de los noventa, aquellos que no creían que lo que estaba ocurriendo era una revolución social sin precedentes (para la que se acuñaron inmediatamente epítetos innumerables: sociedad de la información, sociedad del conocimiento, sociedad Red, sociedad digital, etc.), quedaban relegados a la posición de incrédulos trasnochados. Internet iba a cambiarlo todo e iba a hacerlo de la noche a la mañana (Almiron, 2000). Antes de estas fechas, muchos otros ya habían fomentado la tesis de una tecnología autónoma, fuera para bien (Marshall McLuhan, 1962) o para mal (Alvin Toffler, 1982).

Lo cierto es que el determinismo tecnológico ha sido rebatido por diversos frentes. Investigaciones recientes (por ejemplo Mackenzie, 1985 y Bijker, 1987; citados por Eduard Aibar) han demostrado que el desarrollo tecnológico está tan influenciado por el contexto social como éste, a su vez, lo está por las nuevas tecnologías. La evolución tecnológica no sigue una línea recta del pasado al futuro ni atiende sólo a consideraciones técnicas. De forma que no siempre el resultado de un desarrollo tecnológico es forzosamente el mejor posible, el más eficiente desde un punto de vista técnico. Hay muchas otras razones que tienen que ver con la evolución tecnológica (intereses empresariales, necesidades o deseos de los usuarios, políticas gubernamentales, perfiles culturales, etc.). Pero es evidente que los logros tecnológicos recientes han seducido a muchas personas que, automáticamente, han visto en ellos un potencial de cambio social imparable.

Paralelamente a lo anterior, junto a la euforia tecnológica determinista con la que abandonamos el siglo $\mathrm{XX}$, se afianza progresivamente una actitud bien distinta, contraria a todo determinismo, en algunos casos crítico con él, en otros simplemente indiferente al mismo (ver por ejemplo: Racionero, 2000; o Bruke y Ornstein, 1995). La tecnología no determina nada porque no hay continuidad histórica en los avances humanos. Efectivamente, el siglo XX se ha caracterizado también por una pérdida paulatina de la confianza en el progreso. Es decir, por un abandono gradual pero irremisible de la confianza en la razón humana y en su capacidad para progresar. Un creciente escepticismo se ha apoderado de muchas personas en lo que respecta a los mayores logros tecnológicos del hombre (escepticismo que en ocasiones es irracional, hay personas que incluso se niegan a creer que el hombre haya puesto los pies en la Luna). Puede decirse que buena parte de la humanidad ha descabalgado a la ciencia y a la tecnología del pedestal en el que 
ella misma las había colocado. Ya no estamos tan seguros que el desarrollo científico-tecnológico pueda cumplir todas las promesas que nos habíamos hecho. Además, muchos dudan que los beneficios de la ciencia y la tecnología compensen los perjuicios potenciales que de sus descubrimientos, y de la aplicación de esos descubrimientos, se deriven o puedan derivarse. Frente a los deterministas tecnológicos, que creen en el progreso tecnológico como fuente de progreso social, hay una proporción creciente de personas que ha dejado de creer en el progreso humano en general.

En realidad, es bastante lógico que por fin hiciéramos descender a la razón de su pedestal. Pero el desprestigio creciente que sufren ciencia y tecnología, el escepticismo iracional, la decepción generalizada, el relativismo con que muchas personas valoran sus logros en un mundo lleno de salvajes contradicciones es algo completamente nuevo. Desde el nacimiento de la ciencia moderna, hace ahora cuatro siglos, nunca ésta había pasado por un momento de descrédito como el actual. Entiéndase aquí por descrédito que una proporción creciente de la población duda de que los beneficios del avance científico-tecnológico sean superiores a sus perjuicios o que, en todo caso, a pesar de que sus beneficios sean superiores a sus perjuicios, duda de que sus progresos sean suficientes para eliminar la mediocridad y mezquindad humanas, esto es, los frenos que nos impiden avanzar de verdad. De modo que los pasos hacia adelante quedan en meros progresos cuantitativos, no alcanzan a perfeccionar al individuo como ser pensante.

No deja de ser paradójico que sea precisamente en momentos de grandes éxitos de la racionalidad científica y de su aplicación cuando hagan aparición comportamientos irracionales inexplicables a la luz de todo el conocimiento empírico adquirido y acumulado por la humanidad. Es como si el progreso intelectual acumulado no ejerciera influjo alguno sobre muchas personas. El culto a la vulgaridad crece al tiempo que la cultura pierde progresivamente valor, el relativismo se apodera de forma irracional de la cotidianiedad confundiendo la bondad del "dudar de todo" como a priori filosófico con el "todo vale" de los ignorantes y demagogos. En una era de ubicuidad de la matemática aplicada (qué otra cosa es si no la informática) la magia renace por doquier y el misticismo se apodera de los mismos usuarios de unas máquinas a las que otorgan comportamientos y actitudes esotéricos. La idealización de la juventud, la belleza y la acumulación de riqueza no sólo no decae sino que se convierte en meta perenne junto al irracional triunfo rápido (la obsesión por llegar los primeros sin tener que correr toda la carrera). Y la peor de las paradojas es, sin duda, el aumento de la ignorancia en una sociedad que se llama así misma "del conocimiento". La alergia creciente del ser humano al esfuerzo dilatado y constante (la antítesis del triunfo rápido) deriva hacia una sociedad en la que la mayoría de sus miembros no puede, no quiere o no tiene tiempo para pensar. Y los que todavía lo hacen, acostumbran a dedicar esfuerzos inconmensurables a tareas cuyo valor moral es muy discutible. La élite 
pensante de la sociedad, aquella que realmente la hace evolucionar, es cada día más elitista. Esto es, cada vez hay menos personas, en términos comparativos, que sean significativas para el progreso cualitativo de la especie.

\section{Contradicciones para una era de ¿decadencia o progreso?}

A pesar de todo, hay muestras abundantes de que la fe en el progreso no ha abandonado del todo ni siquiera a los más pesimistas, ¿acaso no es creer en el progreso la adoración constante que, de forma consciente o inconsciente, la mayoría de nosotros siente de lo nuevo por lo nuevo? (adoramos lo nuevo simplemente porque es lo último, asumiendo que lo último siempre será mejor que lo anterior). Pero aunque busquemos una interpretación alejada de los radicalismos de los apologetas del determinismo tecnológico positivo, de los apocalípticos tecnófobos y de los desencantados de la especie humana, las cuestiones que se nos plantean siguen exigiendo respuestas contundentes: ¿La revolución tecnológico-digital que se está produciendo en estos momentos se traducirá en una revolución social? ¿Acaso la irracionalidad creciente se apoderará de la sociedad? ¿Estamos, en suma, en la senda del progreso o de la decadencia, entendiendo a ésta como declive, retroceso con respecto a la situación moral alcanzada en el pasado?

Hay una evidencia palmaria: la explosión está aquí y, aunque desconozcamos cual pueda ser su verdadero alcance, hay un reconocimiento unánime a su peso material. Esto es, la última revolución informacional -la de las telecomunicaciones, Internet, la ingeniería genética, los medios de comunicación y la microinformática- es un salto cuantitativo hacia delante sin precedentes. No hace falta que sigamos glosando sus maravillas técnicas presentes o potenciales. La realidad en términos cuantitativos es flagrante: estamos rodeados de evidencias de la existencia de progreso material, esencialmente tecnológico-digital. Ahora bien, no es fácil emitir un juicio al respecto del valor cualitativo del mismo, esto es, de su impacto en la vida moral de los individuos y, lo más importante, a este salto material sobre el que hay unanimidad se le asocian unas promesas cualitativas de orden social que, sean o no ciertas, se vayan a cumplir o no, chocan frontalmente con algunas de las tendencias más acusadas en la sociedad actual.

Estas tendencias están en abierta contradicción con el acerbo cultural forjado en Occidente y que, se supone, la nueva sociedad surgida de la revolución tecnológico-digital, debería reforzar. Nuestro corpus común de valores democráticos (libertad, igualdad, justicia, educación, convivencia pacífica, etc.) constituyen nuestros objetivos, aquello por lo que medimos el avance social, el progreso en definitiva. Sin ellos, puede existir avance pero sólo será material, esto es, superficial. Para alcanzar estos objetivos podemos utilizar diferentes caminos pero aquel que más pasiones ha suscitado en Occidente es el del crecimiento económico. Y el crecimiento económico, globalizado como dogma de fe debido al poder 
que se le atribuye, se ve acelerado notablemente gracias a la revolución científicotecnológico-digital. De modo que el presupuesto de partida es que esta nueva explosión informacional debería servir a los objetivos citados, esto es, debería aportar no sólo progreso cuantitativo sino también cualitativo, progreso en el ámbito de los valores de las personas.

Pero la irracionalidad creciente en buena parte de nuestros actos contradice buena parte de esos valores. Es como si apuntáramos en una dirección pero corriéramos en otra. Por ello, reflexionar sobre estas contradicciones se hace imprescindible para responder a la pregunta de si lo que nos espera, en esta era de grandes logros materiales, es el progreso o la decadencia moral. Contradiciones como el auge de la irracionalidad, la superficialidad dominante, el relativismo exacerbado, la estupidez o imbecilamiento creciente (esto es, volvernos cada vez más imbéciles), la irresponsabilidad de la inmadurez, el subjetivismo ciego, el egoísmo lacerante y la simplicidad supina en una era de máxima complejidad. Todas ellas en frontal oposición con lo que se presupone debería nacer de una era de la información y el conocimiento: inteligencia y humanidad civilizada. Ahora bien, no podemos olvidar que el poder científico-tecnológico-digital no es algo que se geste al margen de nuestras vidas, exógeno a ellas y cuyo hipotético impacto social no dependa de nuestros propios actos. Así que, probablemente, la respuesta a la pregunta de si avanzamos o retrocedemos a nivel moral con la actual explosión informacional sólo esté en nuestras voluntades. De ahí la dificultad en valorar el actual progreso y la disyuntiva que éste genera. Porque la realidad es tan compleja como lo son las personas que la generan.

\section{Bibliografía:}

- AIBAR, Eduard (2001): "Fatalisme i tecnologia: és autònom el desenvolupament tecnològic?". Internet: http://www.uoc.edu/web/cat/art/uoc/0107026/ aibar.html.

- ALMIRON, Núria (2000): Cibermillonarios. La Burbuja de Internet en Espa$\tilde{n} a$. Barcelona: Planeta.

- BIJKER, W.E.; HUGHES, T.P; PINCH,T. eds. (1987): The Social Construction of Technological Systems: New Directions in the Sociology and History of Technology. Cambridge (MA): MIT Press.

- BRIGGS, Asa y BURKE, Peter (2002): De Gutenberg a Internet. Una historia social de los medios de comunicación. Madrid: Taurus.

- BURKE, James y ORNSTEIN, Robert (1995): Del hacha al chip. Cómo la tecnología cambia nuestras mentes. Barcelona: Planeta Divulgación.

- CASTELLS, Manuel (1997): La era de la información. La sociedad red (vol. I). Madrid: Alianza Editorial. 
- DÍEZ, José A. y ULISES MOULINE, C. (1999). Fundamentos de filosofía de la ciencia. Barcelona: Ariel Filosofía.

- GRAHAM, Gordon (2001). Internet. Una indagación filosófica. Madrid: Frónesis, Cátedra Universitat de València.

- HABERMAS, Jürguen (1988): La lógica de las ciencias sociales. (Madrid: Tecnos, 2002).

- HABERMAS, Jürgen (2001): El futuro de la naturaleza humana. ¿Hacia una eugenesia liberal?. Barcelona: Paidós.

- HAYMAN, Ronald (1980): Nietzsche. A critical Life. Londres: Phoenix Giants.

- KAHN, Herman, WIENER, Anthony y otros (1967): The Year 2000. A framework for speculation on the next thirty-three years. Nueva York: Macmillan.

- LÉVY, Bernard-Henri (2000): El siglo de Sartre. Barcelona: Ediciones B.

- MACKENZIE, D.; WAJCMAN, J. eds.(1985): The Social Shaping of Technology. Buckingham: Open University Press.

- MCLUHAN, Marshall (1962): La galaxia Gutenberg. (Barcelona: Círculo de Lectores, Galaxia Gutenberg, 1993).

- NISBET, Robert (1980): Historia de la idea de progreso. (Barcelona: Gedisa, 1998).

- POPPER, Karl R. (1962). La lógica de la investigación científica. (Madrid: Tecnos, 2001)

- RACIONERO, Luis (2000): El progreso decadente. Madrid: Espasa Calpe.

- ROTHSCHILD, Michael (1990): La bionomía: economía como ecosistema. Internet: htttp://www.bionomics.org/text/spanish/toc.htm.

- TOFFLER, Alvin (1982): La tercera ola. Barcelona: Plaza y Janés. 